\title{
Erratum to: Neurogenic Bladder Secondary to Congenital Spinal Lesions
}

Julia B. Finkelstein ${ }^{1} \cdot$ Lisa Creelman ${ }^{1} \cdot$ Shumyle Alam $^{1}$

Published online: 6 August 2015

(C) Springer Science+Business Media New York 2015

Erratum to: Curr Bladder Dysfunct Rep (2015)

DOI 10.1007/s11884-015-0307-6

The original version of this article unfortunately contained a mistake: Julia B. Finkelstein's surname was incorrectly presented as Finklestein.

The online version of the original article can be found at http://dx.doi.org/ 10.1007/s11884-015-0307-6.

$\triangle$ Shumyle Alam

sa3168@cumc.columbia.edu

1 Division of Pediatric Urology, Columbia UniversityMedical Center, New York Presbyterian Morgan Stanley Children's Hospital, New

York, NY, USA 